\title{
Research on Trust Degree Calculation Model Based on Interval Intuitionistic Fuzzy Sets
}

\author{
Xiaolan Xie and Xiaofeng $\mathrm{Gu}^{*}$ \\ College of Information Science and Engineering, Guilin University Of Technology, Guilin ,Guangxi Zhuang Autonomous Region, \\ China, 541004 \\ ${ }^{*}$ Corresponding author
}

\begin{abstract}
Based on the analysis of the inter - interval intuitionistic fuzzy sets and the interval - valued intuitionistic fuzzy sets, the multi - attribute group decision - making is used to reconstruct the model and apply it to the trust degree calculation of the trust mechanism. By comparing the multiple attributes of trust degree, the attribute weight calculation model is established. Through this model, the multi-attribute aggregation calculation and sorting of trust degree, indirect trust degree, trustworthiness and trust attenuation are obtained. Finally, Method is more comprehensive with the credibility of the region's optimal solution.
\end{abstract}

Keywords-interval intuitionistic fuzzy sets; multi-attribute group decision; trust; trust calculation

\section{INTRODUCTION}

In recent years in a variety of cloud environment, the construction of network platform in the information security issues gradually attention. An indicator of whether information is safe or not is the information we need, data, and resources and resources, resources and users, users and users of the trust between the problem. The results of the trust assessment can visually express whether the information in the information exchange is trustworthy. In the process of trust assessment, many scholars use different theoretical models to study the trust assessment method, the process of trust assessment is a process of trust decision-making, one of the decisive factors of trust decision-making is trust, through the calculation of the trust of the results obtained to make a comprehensive assessment of its trust. Although there are a lot of trust calculation method, but for the trust of such a multi-attribute comprehensive measure of the body is not very good to complete a more comprehensive degree of trust calculation considerations, also can not better describe the trust decision-making state, Therefore, this paper will use the range of intuitionistic fuzzy fuzzy sets of multi-attribute group decision-making algorithm to IVIFS (interval intuitionistic fuzzy sets) to the trust model to establish the relevant model to avoid the information obtained by the calculation process is incomplete or the property itself is fuzzy Uncertainty, etc., and it is difficult to give a precise preference for information.

\section{THE BASIC CONCEPT AND THEORY OF INTERVAL INTUITIONISTIC FUZZY SETS}

\section{A. The Basic Theory of Interval Intuitionistic Fuzzy Sets \\ Intuitionistic fuzzy sets are a kind of expansion and development of traditional fuzzy sets, as a generalization of}

intuitionistic fuzzy sets, interval intuitionistic fuzzy sets are more suitable for dealing with fuzzy information. Here we give the definition of interval intuitionistic fuzzy sets:

Definition 2.1 Let $\mathrm{X}$ be a given nonempty finite domain, the representation of the interval intuitionistic fuzzy sets on $\mathrm{X}$ is:

$$
\left.A=\left\{<x, u_{A}(x), v_{A}(x)>\mid x \in X\right\}=\left\{<x,\left[\overline{u_{A}}(x), \mu_{A}^{+}(x)\right]\left[\bar{v}_{A}(x), v_{A}^{+}(x)\right]\right\rangle x \in X\right\}
$$

Among them, $u_{A}(x), v_{A}(x)$ which represent the membership interval and the non-membership interval of the element $\mathrm{x}$ in the domain $\mathrm{X}$ respectively, and, for any element $\mathrm{x}$ in $\mathrm{x}$, the condition is satisfied: $u_{A}^{+}(x)+v_{A}^{+}(x) \leq 1$ and $u_{A}^{-}(x), v_{A}^{-}(x) \subseteq[0,1]$. For given $x \in X$, $\pi_{A}(x)=\left[1-u_{A}^{+}(x)-v_{A}^{+}(x), 1-u_{A}^{-}(x)-v_{A}^{-}(x)\right]$ is called the "hesitant interval" of interval intuitionistic fuzzy sets.

For convenience, We use IVIFS (X) to denote a set of all the interval intuitionistic fuzzy sets on the domain $\mathrm{X}$. Called the interval interval (abbreviated as $([a, b],[c, d])$ ), which is the "interval intuitionistic fuzzy number", which consists of the subordinate and non-membership of the element $\mathrm{x}$ belonging to $\mathrm{A}$ in $\mathrm{X}$.

In simple terms, the multi-attribute decision-making problem is based on the comprehensive evaluation of multiple attributes of the scheme and then the selection or sequencing of the scheme. Given a multi-attribute decision-making problem, we set the scheme set of $\mathrm{m}$ schemes, and each scheme needs to judge its merits from $n$ attributes. Due to the limitedness and ambiguity of the decision maker's cognition, we use the interval intuitionistic fuzzy number to express the satisfaction and dissatisfaction of the decision maker's concept of "excellent".

Suppose interval fuzzy set decision matrices

$$
R=\left(\left\lfloor a_{i j}, b_{i j}\right\rfloor\left\lfloor c_{i j}, d_{i j}\right\rfloor\right), i \in M, j \in N
$$

$\left\lfloor a_{i j}, b_{i j}\right\rfloor$ indicates that the decision maker $X_{i}$ considers the degree of the scheme to satisfy the attribute $A_{i}$ under the fuzzy concept "excellent"; $\left.\mid c_{i j}, d_{i j}\right\rfloor$ means that decision maker 
$X_{i}$ considers that program $A_{i}$ does not satisfy the degree of attribute in the fuzzy concept "excellent". And there are $\left\lfloor a_{i j}, b_{i j}\right\rfloor \in D[0,1], \quad\left\lfloor c_{i j}, d_{i j}\right\rfloor \in D[0,1], \quad b_{i j}+d_{i j} \leq 1$, $i=1,2, \ldots m, j=1,2, \ldots n$. $A_{i}$.

\section{Establishment of TRUSt DegReE CALCUlation MODEL BASED ON INTERVAL INTUITIONISTIC FUZZY SETS}

\section{A. Common Trust Calculation Method and Decision Method}

Trust measurement refers to how to use mathematical methods to describe the size of the credibility. At present, the main methods are: discrete trust, probability trust, fuzzy trust, trust cloud. Trustworthiness measure is the evaluation of the expected level of the target entity, which is the dynamic variable related to the entity interaction, and has the characteristics of subjectivity, dynamic uncertainty and time lag.

\section{(a) The weighting method}

In the multi-attribute decision-making problem, weighting is divided into decision maker weighting and attribute weighting. Decision-maker weighting mainly includes subjective, objective and combination of three empowerment methods. Attribute weighting is based on the source of data, which is divided into subjective weighting method, objective weighting method and subjective and objective weighting method.

\section{(b) Based on fuzzy mathematics}

Based on the main features of the fuzzy mathematics trust model, the use of natural language to describe the trust, relying on the definition of the function to reflect the degree of trust.

\section{(c) Based on the cloud model approach}

The trust algorithm using cloud model is to input the evaluation value of trust into the inverse cloud algorithm, and the expected expectation is transformed into trust degree. Entropy and super entropy are used to reflect the uncertainty.

For the trust decision-making in terms of the trust of the node, you can use trust decisions to decide whether to interact with some nodes. At present, the more common decision-making methods are the following two kinds:

(a) Set threshold: For the server node, only by the service node to achieve the credibility of the threshold set to provide services. For a serving node, if more than one server node has reached the set threshold, select one of the most trusted servers.

(b) Set trust level: Because trust has the characteristics of subjectivity and uncertainty, trust information can be qualitatively evaluated by natural language, such as: very safe, safe, unsafe, etc., the trust is mapped to a certain trust level through the transformation, and then the trusted decision is made according to the trust policy of the node.

\section{B. Establishment of Trust Degree Calculation Model Based on Interval Intuitionistic Fuzzy Sets}

Trust as an important assessment of the importance of the decision-making indicators, which itself has multiple attributes, For example, we must calculate the trust in the calculation of its direct trust, indirect trust, recommended trust, trust attenuation function finally come to a comprehensive trust. Which direct trust, indirect trust, recommended trust, etc. can be seen as a number of trust attributes. We according to different attributes of different weights for decision-making, and then sort optimization, and ultimately get a comprehensive trust. The following is based on the interval of intuitionistic fuzzy sets of trust calculation technology roadmap:

Trust Calculation Based on Interval Intuitionistic Fuzzy Sets

\section{Trust}

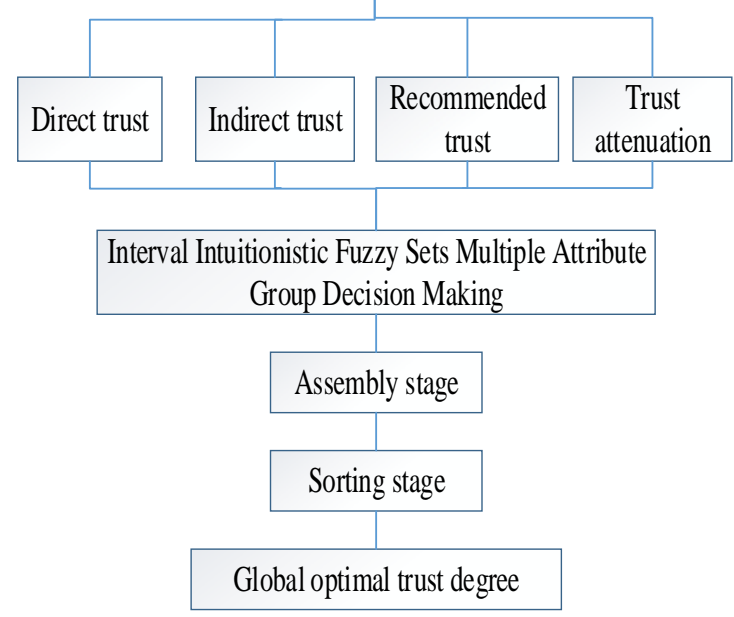

Here we set the direct trust for the property $C_{1}$, indirect trust is attribute $C_{2}$, The recommended trust is attribute $C_{3}$, The trust level is reduced to attribute $C_{4}$, Establish the following trust degree multi-attribute decision table:

TABLE I. TRUST MULTIPLE ATTRIBUTE DECISION TABLE

\begin{tabular}{l|l|l|l|l} 
& $a_{1}$ & $a_{2}$ & $a_{3}$ & $a_{4}$ \\
\hline$C_{1}$ & $x_{11}$ & $x_{12}$ & $x_{13}$ & $x_{14}$ \\
\hline$C_{2}$ & $x_{21}$ & $x_{22}$ & $x_{23}$ & $x_{24}$ \\
\hline$C_{3}$ & $x_{31}$ & $x_{32}$ & $x_{33}$ & $x_{34}$ \\
\hline$C_{4}$ & $x_{41}$ & $x_{42}$ & $x_{43}$ & $x_{44}$
\end{tabular}

Among them, $A=\left\{a_{1}, a_{2}, a_{3}, a_{4}\right\}$ is program set, $x_{i j}$ represents the evaluation value of the attribute $a_{j} \in A(j=1,2 \cdots, n) \quad$ on the attribute $c_{i} \in C(i=1,2 \cdots m)$ (which is the attribute value).

We do not understand the uncertainty of the fuzzy information attribute value for the untrusted trust, the attribute value is interval intuitionistic fuzzy set and the decision maker has the subjective preference, the preference value is a multi attribute problem of interval intuitionistic fuzzy number, that is, 
from a single attribute value to consider, the establishment of each program to achieve their optimal value of the multi-objective planning model to calculate the local program of the best local value, then, from the global consideration, the model of the shortest distance between the local optimal attribute value and the global attribute value of each scheme is established, the comprehensive attribute value of the scheme is calculated, the scheme is sorted or selected. The following trust degree calculation model is established by describing and analyzing the problem of interval intuitionistic fuzzy multiple attribute decision making. First consider from the local attribute value to solve the optimal comprehensive satisfaction of a single scheme $X_{i}(i \in M)$, establish the following two optimal models:

$$
\begin{aligned}
& \text { (Z1) } \max \left\{Z_{i}^{L}=\sum_{j=1}^{n} \xi_{i j} \omega_{j}\right\} \\
& \text { s.t. }\left\{\begin{array}{l}
a_{i j} \leq \xi_{i j} \leq 1-d_{i j}, i=1,2, \cdots, m, j=1,2, \cdots, n \\
\omega=\left(\omega_{1}, \omega_{2}, \cdots \omega_{n}\right)^{T} \in H \\
\sum_{j=1}^{n} \omega_{j}=1 \\
0 \leq \omega_{j} \leq 1, j=1,2, \cdots, n
\end{array}\right. \\
& i=1,2, \cdots, m \text {; } \\
& (Z 2) \max \left\{Z_{i}^{U}=\sum_{j=1}^{n} \eta_{i j} \omega_{j}\right\} \\
& \text { s.t. }\left\{\begin{array}{l}
b_{i j} \leq \eta_{i j} \leq 1-c_{i j}, i=1,2, \cdots, m, j=1,2, \cdots, n \\
\omega=\left(\omega_{1}, \omega_{2}, \cdots \omega_{n}\right)^{T} \in H \\
\sum_{j=1}^{n} \omega_{j}=1 \\
0 \leq \omega_{j} \leq 1, j=1,2, \cdots, n
\end{array}\right. \\
& i=1,2, \cdots, m \text {; }
\end{aligned}
$$

Observe the model, through the analysis of the following two linear programming model:

$$
\begin{array}{r}
(Z 3) \min \left\{Z_{i}^{L L}=\sum_{j=1}^{n} a_{i j} \omega_{j}\right\} \\
\text { s.t. }\left\{\begin{array}{l}
\omega=\left(\omega_{1}, \omega_{2}, \cdots \omega_{n}\right)^{T} \in H \\
\sum_{j=1}^{n} \omega_{j}=1 \\
0 \leq \omega_{j} \leq 1, j=1,2, \cdots, n
\end{array}\right.
\end{array}
$$

$$
\begin{gathered}
\text { (Z4) } \max \left\{Z_{i}^{L U}=\sum_{j=1}^{n}\left(1-d_{i j}\right) \omega_{j}\right\} \\
\text { s.t. }\left\{\begin{array}{l}
\omega=\left(\omega_{1}, \omega_{2}, \cdots \omega_{n}\right)^{T} \in H \\
\sum_{j=1}^{n} \omega_{j}=1 \\
0 \leq \omega_{j} \leq 1, j=1,2, \cdots, n
\end{array}\right.
\end{gathered}
$$

Because the constraints of the two models are the same, after the simple transformation (M3) and (M4), the following models are available:

$$
\begin{gathered}
\text { (Z5) } \max \left\{Z_{i}^{L}=\sum_{j=1}^{n}\left(1-a_{i j}-d_{i j}\right) \omega_{j}\right\} \\
\text { s.t. }\left\{\begin{array}{l}
\omega=\left(\omega_{1}, \omega_{2}, \cdots \omega_{n}\right)^{T} \in H \\
\sum_{j=1}^{n} \omega_{j}=1 \\
0 \leq \omega_{j} \leq 1, j=1,2, \cdots, n
\end{array}\right.
\end{gathered}
$$

According to the same idea analysis model (M2), the following optimization model can be obtained:

$$
\begin{gathered}
\text { (Z6) } \max \left\{Z_{i}^{U}=\sum_{j=1}^{n}\left(1-b_{i j}-c_{i j}\right) \omega_{j}\right\} \\
\text { s.t. }\left\{\begin{array}{l}
\omega=\left(\omega_{1}, \omega_{2}, \cdots \omega_{n}\right)^{T} \in H \\
\sum_{j=1}^{n} \omega_{j}=1 \\
0 \leq \omega_{j} \leq 1, j=1,2, \cdots, n
\end{array}\right.
\end{gathered}
$$

Taking into account the constraints of model (M5) and model (M6), the two models are combined to obtain the following model:

$$
\begin{aligned}
& (Z 7) \max \left\{Z_{i}=\sum_{j=1}^{n}\left(2-a_{i j}-d_{i j}-b_{i j}-c_{i j}\right) \omega_{j}\right\} \\
& i=1,2, \cdots, m
\end{aligned}
$$

Solve the model (M7) to obtain $\varpi_{i}=\left(\varpi_{i 1}, \varpi_{i 2}, \cdots \varpi_{i n}\right)^{T}$, $i=1,2 \cdots, m$, that is the optimal weight of a single scheme, and the optimal satisfaction of a single attribute scheme of trust is as follows:

and 


$$
\begin{aligned}
& \tilde{Z}=\left(\left[\sum_{j=1}^{n} a_{i j} \tilde{\omega}_{i j}, \sum_{j=1}^{n} b_{i j} \tilde{\omega}_{i j},\right],\left[1-\sum_{j=1}^{n}\left(1-c_{i j}\right) \tilde{\omega}_{i j}, 1-\sum_{j=1}^{n}\left(1-d_{i j}\right) \tilde{\omega}_{i j}\right]\right) \\
& =\left(\left[\sum_{j=1}^{n} a_{i j} \tilde{\omega}_{i j}, \sum_{j=1}^{n} b_{i j} \tilde{\omega}_{i j},\right],\left[\sum_{j=1}^{n} c_{i j} \tilde{\omega}_{i j}, \sum_{j=1}^{n} d_{i j} \tilde{\omega}_{i j}\right]\right), i=1,2 \cdots m
\end{aligned}
$$

Secondly, from the global consideration, for the multi-attribute decision-making problem, attribute weight only in the only case, in order to comprehensive evaluation of the program. Next, solve the global optimal weight. Because the decision-making program is fair competition, the decision-maker naturally hopes that the satisfaction of each scheme under the comprehensive attribute weight and the local best satisfaction degree are the smallest, and the deviation function is introduced:

$$
\tilde{d}_{i}=d_{i}\left(\tilde{Z}_{i}, Z_{i}(\omega)\right), i=1,2, \cdots, m
$$

By definition, we can get:

$$
\tilde{d}_{i}=\frac{\left|\sum_{j=1}^{n} a_{i j}\left(\tilde{\omega}_{i j}-\omega_{j}\right)\right|+\left|\sum_{j=1}^{n} b_{i j}\left(\tilde{\omega}_{i j}-\omega_{j}\right)\right|+\left|\sum_{j=1}^{n} c_{i j}\left(\tilde{\omega}_{i j}-\omega_{j}\right)\right|+\left|\sum_{j=1}^{n} d_{i j}\left(\tilde{\omega}_{i j}-\omega_{j}\right)\right|}{4}
$$

According to the smaller the better the principle of deviation, we can establish the following trust degree of multi-attribute optimization model:

$$
\begin{gathered}
(Z 8) \min \tilde{d}_{i}=d_{i}\left(\tilde{Z}_{i}, Z_{i}(\omega)\right) \\
\text { s.t. }\left\{\begin{array}{l}
\omega=\left(\omega_{1}, \omega_{2}, \cdots, \omega_{n}\right)^{T} \in H \\
\sum_{j=1}^{n} \omega_{j}=1 \\
0 \leq \omega_{j} \leq 1, i=1,2, \cdots, m, j=1,2, \cdots, n
\end{array}\right.
\end{gathered}
$$

The above model can be transformed into a single trust planning model with integrated trust, as follows:

$$
\begin{gathered}
(Z 9) \min =\sum_{i=1}^{m} \tilde{d}_{i} \\
\text { s.t. }\left\{\begin{array}{l}
\left|\sum_{j=1}^{n} a_{i j}\left(\tilde{\omega}_{i j}-\omega_{j}\right)\right|+\left|\sum_{j=1}^{n} b_{i j}\left(\tilde{\omega}_{i j}-\omega_{j}\right)\right|+\left|\sum_{j=1}^{n} c_{i j}\left(\tilde{\omega}_{i j}-\omega_{j}\right)\right|+\left|\sum_{j=1}^{n} d_{i j}\left(\tilde{\omega}_{i j}-\omega_{j}\right)\right|-4 \tilde{d}_{i}=0 \\
\tilde{d}_{i} \geq 0, i=1,2, \cdots, m \\
0 \leq \omega_{j} \leq 1, j=1,2, \cdots, n
\end{array}\right.
\end{gathered}
$$

The global optimal fitness weight vector, global best satisfaction, can be obtained by solving the model. Finally, the interval of intuitionistic fuzzy numbers is obtained based on the idea of possibility, so as to obtain the optimal comprehensive trust degree.

\section{CONCLUSION}

Trust as a decisive factor in trust decision-making, information security, information and other aspects of trust is an important consideration, although there are a lot of trust calculation methods, but for the trust of such a multi-attribute synthesis of the body is not very good to complete a more comprehensive confidence in the calculation of domain considerations, can not better describe the trust decision-making state, therefore, we study the IVIFS (interval intuitionistic fuzzy sets) in the multi-attribute group decision algorithm for interval intuitionistic fuzzy sets, It is difficult to give the problem of accurate preference information because of the incomplete information or the fuzzy uncertainty of the attribute itself in the calculation process. Although the content of the article is not particularly substantial, but in the next period, we will continue to study hard to make more rich research results.

\section{REFERENCES}

[1] Xu Z.S. Maximum Deviation Method Based on Deviation Degree and Possibility Degree for Uncertain Multi-attribute Decision Making [J]. Control and Decision, 2011,16:818- 821.

[2] Zhang Y J, Ma P J, Su X H. Zhang C P. Interval Intuitionistic Fuzzy Multiple Attribute Decision Making under uncertainty attribute weights [J].Journal of Automation, 2012, 38(2):220-228.

[3] J W. Robust Optimization Analysis for Multiple Attribute Decision Making Problems with Imprecise Information [J]. Annals of Operations Research, 2012, 197:109-122.

[4] Xu Yejun, Wang Huimin, Palacios-Marques, Daniel. An Interactive Approach Based on Alternative Achievement Scale and Alternative Comprehensive Scale for Multiple Attribute Decision Making under Linguistic Environment [J], International Journal of Computational Intelligence Systems,2013,6(1):87-95.

[5] Yue Z, Jia Y. An application of soft computing technique in group decision making under interval-valued intuitionistic fuzzy environment[J]. Applied Soft Computing, 2013, 13(5): 2490-2503.

[6] Wei Guiwu, Wang Hongjun, Zhao Xiaofei, Lin Rui. Approaches to hesitant fuzzy multiple attribute decision making with incomplete weight information [J], Journal of Intelligent and Fuzzy Systems, 2014, 26(1) :259-266.

[7] Liu B S, Shen $Y$ H, Chen X H, et al. A complex multi-attribute large-group PLS decision-making method in the interval-valued intuitionistic fuzzy environment[J]. Applied Mathematical Modelling, 2014, 38: 4512-4527.

[8] Jin F F, Pei L D, Chen H Y et al. Interval-valued intuitionistic fuzzy continuous weighted entropy and its application to multi-criteria fuzzy group decision making[J]. Knowledge-Based Systems, 2014, 59: 132-141.

[9] Zhang X, Xu Z. Soft computing based on maximizing consensus and fuzzy TOPSIS approach to interval-valued intuitionistic fuzzy group decision making[J]. Applied Soft Computing, 2015,26: 42-56.

[10] Joshi D, Kumar S. Interval-valued intuitionistic hesitant fuzzy Choquet integral based TOPSIS method for multi-criteria group decision making[J]. European Journal of Operational Research, 2016, 248(1): 183-191. 